\section{Origins of tife}

\section{A left-handed Solar System?}

\section{Christopher F. Chyba}

$\square$ ow and why did life on Earth come to use almost exclusively laevorotatory, or left-handed amino acids (L-enantiomers), rather than their mirror-image dextrorotatory, or right-handed forms (Denantiomers)? The paper by Engel and Macko on page 265 of this issue ${ }^{1}$ re-examines the Murchison meteorite, and reinforces the surprising result that some process favouring L-enantiomers seems to have operated before the origin of life on Earth, and probably before the formation of the Solar System. If this is correct, our Solar System may have formed with a built-in bias for left-handed amino acids.

An excess of L-enantiomers is an intriguing result, as it suggests that the handedness, or chirality, of terrestrial biology might have an extraterrestrial cause. But an L-excess indigenous to the meteorite is difficult to prove, as it may instead be due to contamination by the terrestrial biosphere of an originally racemic mixture. (In a racemic mixture, $\mathrm{L}$ - and $\mathrm{D}$-enantiomers are present in equal abundance, as in typical laboratory chemical syntheses of amino acids.)

When amino acids were first discovered in the Murchison meteorite ${ }^{2}$, apparent excesses of $\mathrm{L}$-enantiomers ranging from 0 to $20 \%$ were found. However, the conservative interpretation of these results was that contamination was to blame, especially as nonprotein amino acids were reported to show no enantiomeric excess ${ }^{3}$. ('Protein' amino acids are of the type used by terrestrial life in proteins, and are therefore the most likely to suffer from biological contamination.) A later study ${ }^{4}$, claiming indigenous enantiomeric excesses in Murchison of up to $70 \%$, was criticized on similar grounds ${ }^{5}$.
This controversy led Pillinger ${ }^{6}$ to emphasize the need for a criterion independent of enantiomeric excess to judge the authenticity of meteoritic amino acids. He suggested determining the isotopic composition of individual enantiomers, because terrestrial stable isotope ratios differ from those in meteorites.

Engel and Macko $^{1}$ appear to have achieved this. These authors had previously ${ }^{7}$ used the carbon isotope ratio ${ }^{13} \mathrm{C} /{ }^{12} \mathrm{C}$ of alanine in Murchison to argue for an excess of L-alanine over D-alanine, but reservations about these measurements persisted ${ }^{8}$. In their present letter ${ }^{1}$, they instead examine the ${ }^{15} \mathrm{~N} /{ }^{14} \mathrm{~N}$ ratio of individual Murchison amino-acid enantiomers. They find L-enantiomer excesses in the amino acids alanine and glutamic acid - both protein amino acids - of over $30 \%$ and $50 \%$, respectively, with ${ }^{15} \mathrm{~N} /{ }^{14} \mathrm{~N}$ ratios that are too high to include much terrestrial material. The contradiction between this and an earlier report ${ }^{2}$ that alanine and glutamic acid have L-enantiomer excesses of about 0 and $10 \%$, respectively, suggests that the Murchison meteorite is very heterogeneous.

Another way to establish an extraterrestrial origin is to use amino acids that are extremely rare in the terrestrial biosphere. This was used by Zhao and Bada ${ }^{9}$ to argue that two amino acids found above and below the 65-million-year-old Cretaceous/Tertiary boundary layer in Stevns Klint, Denmark, were not terrestrial contaminants. The same criterion was recently applied by Cronin and Pizzarello $^{10}$ to the Murchison meteorite. These authors discovered enantiomeric excesses of up to $9 \%$ in apparently non-biological amino acids. So it appears that two very different approaches in two laboratories confirm enantiomeric excesses in the Murchison meteorite.

The same isotope ratios that distinguish Murchison amino acids from their terrestrial counterparts imply that these molecules or their chemical precursors originated in interstellar clouds ${ }^{1,8}$. Why should chemistry in such a cloud have a bias towards onehandedness? One possibility ${ }^{10}$ is that an enantiomeric preference may have been imposed on the cloud out of which our Solar System formed by circularly polarized light, perhaps synchrotron radiation from a neutron star ${ }^{11}$. If a substantial fraction of the organic inventory of early Earth was then derived from comets and asteroids ${ }^{12}$, the synchrotron-radiation hypothesis would connect terrestrial biochemistry with the extreme physics of collapsed stars.

If the excess was indeed established by some process specific to the molecular cloud out of which our Solar System formed, similar enantiomeric excesses should be found in other organic-rich meteorites and in comets. Examination of other carbonaceous chondrites could therefore test this hypothesis. Unless there is a more widespread process operating, other solar systems could have been formed with a preference for D-amino acids, or with no preference at all — with

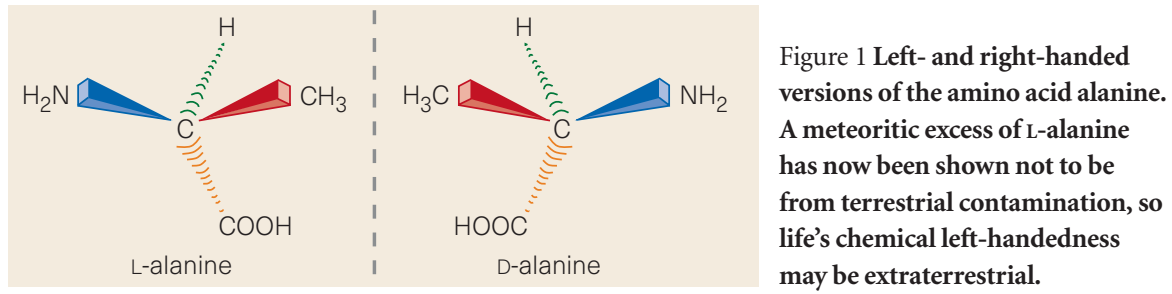

\title{
Avoided objects
}

\section{Freudian abstractS Dust and fibres from Freud's couch}
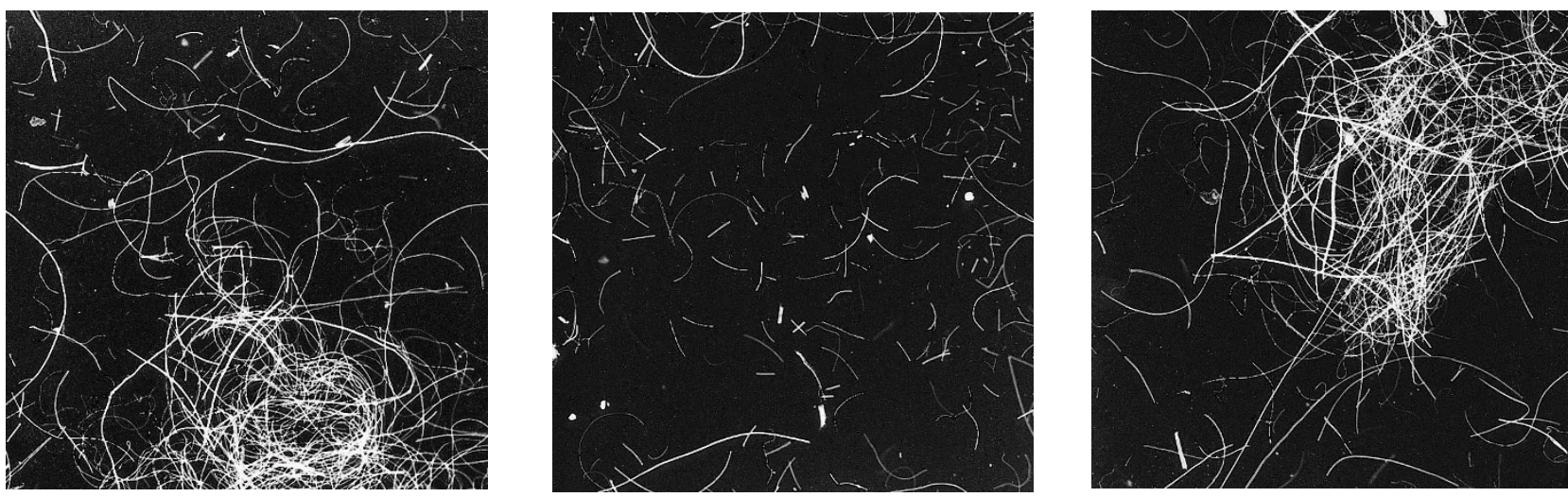
possible consequences for life in those systems. Alternatively, if the enantiomeric excess somehow arose during chemical evolution within Murchison itself, no correlation between meteorites is demanded.

Although these enantiomeric excesses suggest a link between extraterrestrial organic molecules and the origin of terrestrial life, the connection is by no means certain. A preference may have arisen during prebiotic or biotic evolution as well. For example, consider peptide nucleic acid (PNA), a candidate DNA precursor molecule. It has been shown that an otherwise achiral PNA strand can have its chirality fixed by the presence of an Lor D-lysine residue attached to its end ${ }^{13}$. One can imagine a picture in which this random 'seeding' of chiralityled to a chiral preference in either prebiotic chemistry or early life ${ }^{14}$. So even if the Solar System was born with a preference for L-amino acids, there may have been other opportunities for chiral choices to be made, and our understanding is far too limited to know whether these subsequent choices might have dominated any initial enantiomeric bias.

Finally, Lederberg ${ }^{15}$ suggested in 1965 that one criterion for detecting extraterres- trial life in the Solar System might be to search for enantiomeric excesses. Mounting evidence for (evidently non-biological) enantiomeric excesses in the Murchison meteorite means that this criterion alone may be less useful than we had hoped whether within martian meteorites, on the martian surface, or elsewhere.

Christopher F. Chyba is in the Department of Planetary Sciences, University of Arizona, Tucson, Arizona 85721, USA.

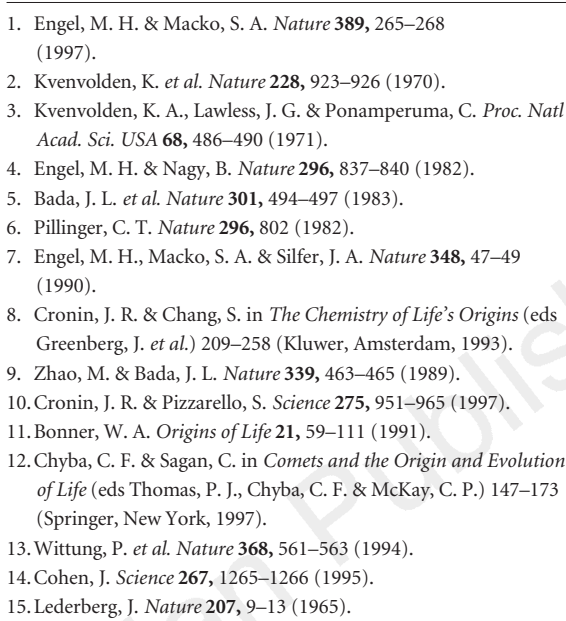

8. Cronin, J. R. \& Chang, S. in The Chemistry of Life's Origins (eds Greenberg, J. et al.) 209-258 (Kluwer, Amsterdam, 1993).

9. Zhao, M. \& Bada, J. L. Nature 339, 463-465 (1989).

10. Cronin, J. R. \& Pizzarello, S. Science 275, 951-965 (1997).

11. Bonner, W. A. Origins of Life 21, 59-111 (1991).

12. Chyba, C. F. \& Sagan, C. in Comets and the Origin and Evolution of Life (eds Thomas, P. J., Chyba, C. F. \& McKay, C. P.) 147-173 (Springer, New York, 1997).

13. Wittung, P. et al. Nature 368, 561-563 (1994).

14. Cohen, J. Science 267, 1265-1266 (1995).

15. Lederberg, J. Nature 207, 9-13 (1965).

$\mathrm{t}$ is remarkable that we perceive a threedimensional world using two-dimensional images. Artists discovered that depth perception arises from perspective, shading and occlusion of distant objects by closer ones. Yet it was not until Wheatstone invented the stereoscope, in 1838 , that it was realized that differences in the positions of images in our two eyes - binocular disparities - also create impressions of depth ${ }^{1}$. (One of Wheatstone's stereograms is shown in Fig. 1.)

Recently, people have become fascinated by autostereograms, in which a threedimensional scene emerges when the eyes misconverge on an apparently random array of dots. We now have stereoscopic virtual reality and stereoscopic 'vision' in robots. But what are the brain mechanisms that are involved in stereopsis (binocular vision) ${ }^{2}$ ? An advance in our understanding of these mechanisms comes from results by Cumming and Parker ${ }^{3}$ and by Masson et al. ${ }^{4}$, on pages 280 and 283, respectively, of this issue.

When we look at a small object, the images of it fall on corresponding points in the two retinas. Neural signals from these corresponding points then converge on the same 'binocular cell' in the primary visual cortex (V1) of the brain ${ }^{5}$. As a result, the images fuse and we see one object. But if the images differ widely in shape, orientation or luminance contrast, they rival one another rather than fuse. When similar images fall on slightly different retinal regions, we see one object standing out in depth (Fig. 1). This is the magic of stereoscopic vision. The simplest idea is that optic-nerve fibres serving slightly different regions (receptive fields) in each of the two retinas converge onto the same binocular cell. Such a cell is a 'disparity detector', because it responds best when the images in the two eyes have a disparity that matches the difference in the positions of the two receptive fields ${ }^{6}$. Different disparity detectors respond to different signs and magnitudes of disparity. Zero-disparity detectors respond to objects in the plane of fixation, crossed-disparity detectors react to nearer objects, and uncrossed-disparity

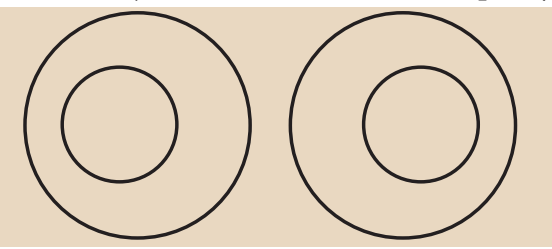

Figure 1 One of the original stereograms constructed by Wheatstone in 1838 . When the displays are fused by convergence, the inner ring appears nearer than the outer ring, because its images have a crossed disparity. When the displays are fused by divergence, the inner ring appears more distant, because its images have an uncrossed disparity. detectors respond to more distant objects. This is a position-disparity mechanism. Disparities may also be detected by differences in the distribution of excitatory and inhibitory regions within two receptive fields that do not differ in position. This is a phase-disparity mechanism.

A simple disparity detector that receives direct inputs from the two eyes does not produce a pure disparity signal - one that is not influenced by incidental changes in the stimulus. For example, the signal for black bars is not the same as that for white bars, and simple detectors are sensitive to slight changes in the location of the object ${ }^{7}$. In the cat, more robust disparity signals are produced by so-called complex cells at a later stage of processing. But, even for some complex detectors, the sign of the disparity signal is inverted when the contrast sign is reversed such that white regions in one eye are superimposed on black regions in the other ${ }^{8}$.

Cumming and Parker $^{3}$ now report that most binocular cells in V1 of the monkey produce a normal disparity signal to images with the same sign of contrast, but an inverted signal to reversed-contrast images. Visually, images with reversed contrast evoke rivalry, but they do not create an impression of depth (see Fig. 1 on page 281). These results are important because they show that disparity detectors in $\mathrm{V} 1$ of the primate respond to a type of disparity that is not used directly for depth perception. Furthermore, they indicate that (contrary to the generally held view), cells in V1 cannot detect when images are in register - this function presumably occurs in a higher visual centre.

What are these inverted-disparity signals used for? Two or more simple detectors could feed into a complex detector which accepts only same-contrast signals. Other complex cells could use reversed-contrast signals to evoke rivalry and help to indicate whether complex images in the two eyes are in proper register (that is, whether they have a maximum proportion of same-contrast edges). But periodic stimuli, even when in register, can contain regions of opposite contrast. Under these circumstances, complex cells that accept opposite-contrast stimuli could use them to improve their response ${ }^{8}$.

When we look at a nearby object our eyes converge, and when we look at a far object our eyes diverge. These eye movements bring the images of the object of interest, as far as possible, onto corresponding points, and simplify the task of detecting disparities due to relative depth. Given that reversed-contrast images produce inverted disparity signals, do they also evoke reversed vergence?

To answer this question, Masson et al. ${ }^{4}$ measured the vergence responses of monkeys and humans to arrays of overlapping reversed-contrast disks. Vergence occurred in the opposite direction to that evoked by same- 